\title{
Differential binding of Shiga toxin 2 to human and murine neutrophils
}

\author{
Thomas P. Griener, ${ }^{1}$ George L. Mulvey, ${ }^{1}$ Paola Marcato ${ }^{2}$ \\ and Glen D. Armstrong ${ }^{1}$
}

Correspondence

Glen D. Armstrong

glen.armstrong@ucalgary.ca

Received 11 March 2007

Accepted 28 June 2007

\author{
${ }^{1}$ Department of Microbiology and Infectious Diseases, University of Calgary Health Sciences \\ Centre, Calgary, Alberta T2N 4N1, Canada \\ ${ }^{2}$ Department of Microbiology and Immunology, Sir Charles Tupper Medical Building, Dalhousie \\ University, Halifax, Nova Scotia B3H 1X5, Canada
}

\begin{abstract}
Shiga toxins (Stx1 and Stx2) are responsible for initiating haemolytic uraemic syndrome, a serious extraintestinal complication caused by enterohaemorrhagic Escherichia coli $\mathrm{O} 157: \mathrm{H} 7$ infection in humans. Shiga toxins are classical $A B_{5}$-type exotoxins, consisting of a globotriaosylceramide $\left(\mathrm{Gb}_{3}\right)$-binding B subunit pentamer and an enzymic A subunit. It is demonstrated in this study that Stx2 binds to human neutrophils by a non-classical mechanism that is independent of $\mathrm{Gb}_{3}$. In contrast, the investigation revealed that $\mathrm{Stx} 2$ binds to murine neutrophils by the classical $\mathrm{Gb}_{3}$ dependent mechanism. Moreover, whereas the human serum amyloid P (HuSAP) component inhibited Stx2 binding to murine neutrophils, HuSAP increased Stx2 binding to human neutrophils by $84.2 \%$ ( $P \leqslant 0.002$, Student's $t$-test). These observations may explain why HuSAP protects mice from the lethal effects of Stx2, whereas there is no indication that HuSAP plays a similar protective role in humans infected by $E$. coli $\mathrm{O} 157: \mathrm{H} 7$.
\end{abstract}

\section{INTRODUCTION}

Enterohaemorrhagic Escherichia coli (EHEC), which include E. coli serotype O157:H7, are associated with the clinical conditions haemorrhagic colitis and occasionally haemolytic uraemic syndrome (HUS) in humans (Karmali et al., 1983; Nataro \& Kaper 1998). HUS is defined by a triad of clinical features, which ultimately impact negatively on kidney function (Kaplan et al., 1998). HUS results from the cytotoxic activity of the Shiga toxins (Stx1 and Stx2), which are expressed by all EHEC serotypes. Shiga toxins consist of a single enzymic A subunit and five identical B subunits assembled into a toroid-shaped pentamer (Fraser et al., 2004), which anchors the holotoxin to the $\alpha \mathrm{Gal}(1,4) \beta \mathrm{Gal}(1,4) \beta \mathrm{Glc}$ glycan sequence of the neutral glycolipid, globotriaosylceramide $\left(\mathrm{Gb}_{3}\right)$ (Liu et al., 1999), on the surface of host cells, thereby initiating delivery of the A subunit to its intracellular target (Cherla et al., 2003).

Although the process whereby Shiga toxins initiate targetcell death is reasonably well established, how the toxins arrive at the extraintestinal target organs is unclear. In this regard, te Loo et al. (2000, 2001a, b) reported that Stx1 and Stx2 bind to the surface of human neutrophils

Abbreviations: AF, Alexa Fluor; EHEC, enterohaemorrhagic Escherichia coli; $\mathrm{Gb}_{3}$, globotriaosylceramide; HI-FBS, heat-inactivated fetal bovine serum; HUS, haemolytic uraemic syndrome; HuSAP, human serum amyloid $\mathrm{P}$ component; $\mathrm{PE}$, phycoerythrin; RFU, relative fluorescent unit. both in vitro and in vivo. These studies also revealed that a significant portion of Stx remained on the surface of human neutrophils and that this binding did not appear to involve conventional $\mathrm{Gb}_{3}$ receptors, which are not expressed by these cells (Macher \& Klock, 1980; Fukuda et al., 1985). It has been inferred from these findings that Stx1 and Stx2 might be transported to their extraintestinal target organs on the surface of circulating neutrophils.

In other studies, we and others have reported that Stx2 binds specifically to the human serum amyloid P (HuSAP) component in blood (Kimura et al., 2001; Marcato et al., 2003). Moreover, HuSAP inhibits the cytotoxic action of Stx2 in vitro (Marcato et al., 2003) and protects mice from this toxin's lethal effects in vivo (Armstrong et al., 2006). Paradoxically, we found no significant correlation between the concentration of HuSAP and the clinical outcome in patients suffering from an E. coli O157:H7 infection, an observation that contrasts with the protective action of HuSAP in mice (Armstrong et al., 2006). These results also confirmed that the binding of Stx1 and Stx2 to the surface of human neutrophils might involve an unconventional mechanism, as it occurs in the presence of HuSAP, which non-competitively inhibits Stx2 binding to $\mathrm{Gb}_{3}$ receptors. Accordingly, the studies described in this paper were conducted to provide further insight into the interactions between Stx2 and HuSAP, as well as the surface of human neutrophils. 


\section{METHODS}

Cell lines. Ramos Burkitt's lymphoma B cells were cultivated in RPMI 1640 (Sigma Aldrich) supplemented with $4.5 \mathrm{mM}$ glucose, $2 \mathrm{mM}$ glutamine, $1 \mathrm{mM}$ sodium pyruvate, $10 \mathrm{mM}$ HEPES and $10 \%$ heat-inactivated fetal bovine serum (HI-FBS). ACHN (CRL-1611) human renal tubular epithelial cells were obtained from the American Type Culture Collection and propagated according to the supplier's instructions.

Protein purification and labelling with Alexa Fluor (AF). Stx1 and Stx2 were expressed and affinity purified using Synsorb Pk as described previously (Mulvey et al., 1998). Endotoxin was removed from the Stx preparations using EndoTrap blue LPS affinity columns (Profos) according to the supplier's instructions. The amount of LPS remaining in these preparations was determined to be $<0.001$ endotoxin units ( $\mu$ g protein $)^{-1}$ as assessed by a Limulus amoebocyte lysate PYROGENT gel clot assay (Cambrex). The Stx2 A subunit and B subunit pentamer were purified by FPLC from the holotoxin preparations as described by Head et al. (1991). SDS-PAGE analysis combined with a sensitive silver staining technique confirmed that these subunit preparations possessed no holotoxin contamination. In addition, minimal $(<0.01 \%)$ holotoxin contamination was detected in the subunit preparations as determined by a Vero cytotoxicity assay (data not shown). Stx1 and Stx2 were labelled with AF 488 carboxylic acid succinimidyl ester (Invitrogen) in $250 \mathrm{mM} \mathrm{NaCl}$ (pH 7.0) while bound to the Synsorb $\mathrm{Pk}$ affinity matrix to preserve their $\mathrm{Gb}_{3}$-binding activity. The cytotoxic activity (50\% cytotoxic dose) of the AFlabelled toxin preparations (denoted Stx1-488 and Stx2-488) was evaluated in a Vero cytotoxicity assay and found to be comparable to that of unlabelled toxin (380 pg per $10^{5}$ cells vs 430 pg per $10^{5}$ cells, respectively). HuSAP was purified as described by Hawkins et al. (1991) and kindly provided by Dr M. B. Pepys (Royal Free \& University College Medical School, University College London, UK).

The Stx subunits as well as HuSAP were AF-labelled in solution according to the manufacturer's instructions. The AF-labelled HuSAP (HuSAP-488) retained its $\mathrm{Ca}^{2+}$-independent Stx2-specific binding capabilities as determined by an ELISA procedure described previously (Armstrong et al., 2006).

Whole-blood binding assays. Blood was collected after obtaining informed consent from healthy human volunteers. An ELISA was used to screen donor sera for immunoreactivity to Stx1 and Stx2, and only blood from seronegative individuals was used in the experiments. Aliquots of blood $(500 \mu \mathrm{l})$ were incubated with either no toxin or $5 \mu$ g purified Stx 1 or Stx 2 at room temperature for $2 \mathrm{~h}$. The cellular and cell-free serum fractions were then separated by centrifuging the blood samples at $100 \mathrm{~g}$ for $5 \mathrm{~min}$. The supernatant was removed and the sedimented blood cells were resuspended in $500 \mu \mathrm{l} \mathrm{PBS}$. A fraction of the cells was applied to glass microscope slides and fixed in situ with methanol. These preparations were incubated at $22{ }^{\circ} \mathrm{C}$ for $1 \mathrm{~h}$ with a 200 -fold dilution of rabbit polyclonal anti-Stx1 or anti-Stx 2 antibody diluted in human serum to inhibit the reaction of the antibodies with $\mathrm{Fc}$ receptors. After rinsing the slides with double-distilled water $\left(\mathrm{ddH}_{2} \mathrm{O}\right)$, the cells were incubated at $22{ }^{\circ} \mathrm{C}$ for $1 \mathrm{~h}$ with a 200 -fold dilution of AF 488 labelled goat anti-rabbit antibody (Invitrogen), in addition to a 100fold dilution of mouse monoclonal phycoerythrin (PE)-labelled anti-CD13 (BD Biosciences) diluted in human serum, for identification of neutrophils. The preparations were rinsed thoroughly with $\mathrm{ddH}_{2} \mathrm{O}$ and analysed by confocal microscopy using a Leica DMIERB2 inverted microscope equipped with a $\times 63$ oil-immersion objective and an Orca ER cooled charge-couple device camera.

Neutrophil isolation. Blood was collected from healthy human donors and erythrocytes were removed using dextran sedimentation
(6\% in PBS) and hypotonic lysis in $\mathrm{ddH}_{2} \mathrm{O}$. The neutrophils were isolated from the resulting cell suspensions using Ficoll-Histopaque (Sigma Aldrich) density centrifugation and assessed by forward- and side-scatter flow cytometric analysis, as well as staining with a fluorescently labelled antibody specific to the neutrophil marker GR1, to be $>95 \%$ viable and $>97.5 \%$ pure. The neutrophils were then suspended in the desired medium and stored on ice until needed. The method and process used for obtaining samples were reviewed by the Ethics Review Panel of the University of Calgary. Murine neutrophils were collected from the marrow fraction obtained from the femurs and tibias of C57BL/6 mice. The marrow was removed by perfusing the bones with ice-cold PBS following resection of the bone ends. A homogeneous suspension of cells was produced by drawing the marrow through a 20-gauge needle and these cells were then sedimented by centrifugation for $12 \mathrm{~min}$ at $4{ }^{\circ} \mathrm{C}$ at $250 \mathrm{~g}$. The resulting cell pellet was suspended in PBS, layered over a discontinuous Percoll (Sigma Aldrich) gradient composed of a stock solution ( $90 \mathrm{ml}$ Percoll, $10 \mathrm{ml} 10 \times$ Hanks' balanced salt solution) diluted to 72,64 and $52 \%$ in Hanks' balanced salt solution. The gradients were centrifuged at $1100 \mathrm{~g}$ for $30 \mathrm{~min}$. The mature murine neutrophil fraction was removed and the cells were washed in PBS and finally suspended in the desired medium. All procedures conformed to the requirements of the Canadian Council on Animal Care and the protocol was approved by the University of Calgary Health Sciences Animal Care Committee.

Cell-binding assays. The AF-labelled proteins were diluted to the desired concentrations in PBS or RPMI $1640+20 \%$ HI-FBS, and then added to $10^{5}$ cells. Binding was allowed to proceed for $30 \mathrm{~min}$ at $37{ }^{\circ} \mathrm{C}$. The cells were then washed, resuspended in buffer and analysed by flow cytometry or confocal microscopy. Binding inhibition assays were performed by pre-incubating $10^{5}$ cells with an excess of the unlabelled protein for $30 \mathrm{~min}$ at $37^{\circ} \mathrm{C}$ prior to adding the AF-labelled protein, or pre-incubating AF-labelled toxin with excess inhibitor [DAISY (a multivalent $\mathrm{Gb}_{3}$ receptor analogue) or HuSAP] prior to addition of cells.

Stx transfer assays. Stx2-488 was incubated with neutrophils suspended in RPMI $1640+10 \%$ HI-FBS for $30 \mathrm{~min}$ at $37{ }^{\circ} \mathrm{C}$ in the presence or absence of HuSAP. The neutrophils were then washed three times to remove any unbound Stx2-488 and HuSAP, and resuspended in either fresh HuSAP-supplemented or HuSAP-free medium containing Ramos cells at the same concentration as the neutrophils. These mixtures were incubated at $24{ }^{\circ} \mathrm{C}$ and sampled at 5 min intervals thereafter. The amount of label transferred from the Stx2-488-loaded neutrophils to the unlabelled Ramos cells in the presence or absence of HuSAP was then determined by flow cytometry analysis in which Ramos and neutrophil cell populations were differentiated by means of forward- and side-scatter measurements with $<5 \%$ signal crossover during the course of the experiment.

\section{RESULTS AND DISCUSSION}

\section{Neutrophil binding by Shiga toxins}

The immunofluorescence images presented in Fig. 1 revealed that both Stx1 and Stx2 bound specifically to polymorphonuclear leukocytes in human whole-blood samples. Although these observations compare favourably with those already published by several groups (te Loo et al., 2000, 2001a, b; Tazzari et al., 2004; Brigotti et al., 2006), they contrast with some reported more recently (Flagler et al., 2007; Geelen et al., 2007). However, in order to isolate neutrophils for analysis, Flagler et al. (2007) 

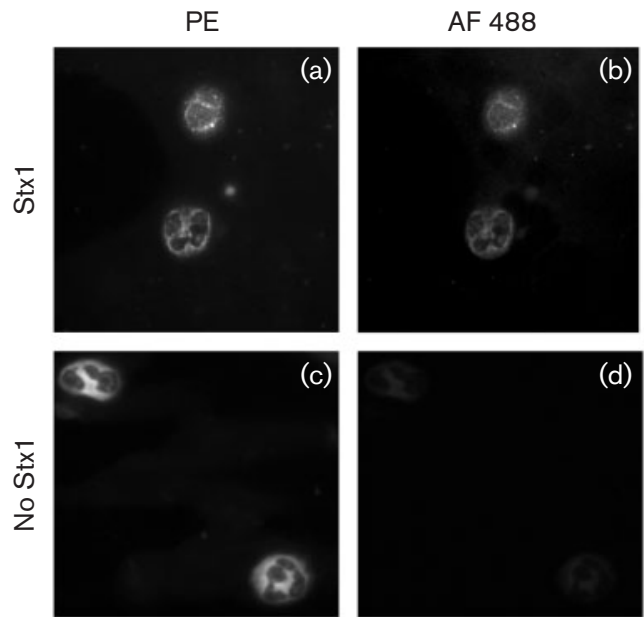
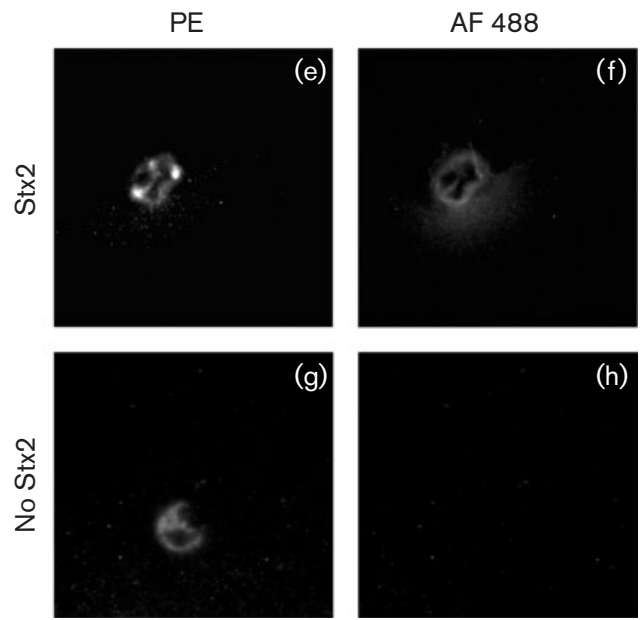

Fig. 1. Immunofluorescence of whole blood cells following a $2 \mathrm{~h}$ incubation with no toxin (c, d, g, h) or $5 \mu$ g Stx 1 (a, b) or Stx2 $(e, f)$. The immobilized toxin-treated cells were then incubated with rabbit polyclonal anti-Stx1 (a-d) or anti-Stx2 (e-h), followed by AF 488-labelled goat anti-rabbit lgG, as well as PE-labelled anti-CD13 to assist in identifying neutrophils. Cells were visualized by confocal microscopy using filters specific for PE fluorescence ( $a, c, e, g)$ or AF 488 fluorescence (b, d, f, h).

subjected their Stx-treated blood samples to a $30 \mathrm{~min}$ centrifugation step that employed Mono-Poly resolving medium. In contrast, the protocol used here involved a 5 min centrifugation step to fractionate the Stx-treated blood into its total cellular and cell-free components. The discrepancy between the results reported by Flagler et al. (2007) and those presented here, as well as those in older studies (te Loo et al., 2000), may possibly be explained by the difference in the time taken to process the blood samples for subsequent analysis. The possible inhibitory effect of Mono-Poly resolving medium on Stx binding to human neutrophils is also an unknown variable. Regardless, the observation of Stx-bearing neutrophils in the circulation of human subjects infected with E. coli O157 : H7 (te Loo et al., 2001b; Tazzari et al., 2004; Brigotti et al., 2006) would strongly suggest that, in vivo, Stx can remain bound to the surface of neutrophils long enough for these to transport the toxins to endothelial cells in the glomerular capillaries and other target sites.

\section{Effect of HuSAP on Stx2 binding to host cells}

Although we previously reported that HuSAP prevented the cytotoxic activity of Stx2 in vitro and in the murine Shiga toxaemia model (Marcato et al., 2003; Armstrong et al., 2006), these investigations did not address the mechanism of this protective action. The data presented in Fig. 2 directly demonstrated that HuSAP inhibits Stx2-488 binding to ACHN human renal tubular epithelial cells, a cell line to which the toxin binds via the $\mathrm{Gb}_{3}$ receptor (Taguchi et al., 1998). As anticipated, as it does not bind to HuSAP (Marcato et al., 2003), Stx1-488 binding to ACHN cells was unaffected by the presence of HuSAP. In contrast to the observations with ACHN cells, HuSAP failed to inhibit Stx2488 binding to isolated human neutrophils (Fig. 3). This is consistent with the observation that Stx2 binds to human neutrophils in whole blood (Fig. 1), which contains HuSAP. In fact, HuSAP increased the binding of Stx2-488 to human neutrophils by $84.2 \%$ (Fig. $3, P<0.002$, Student's $t$-test).
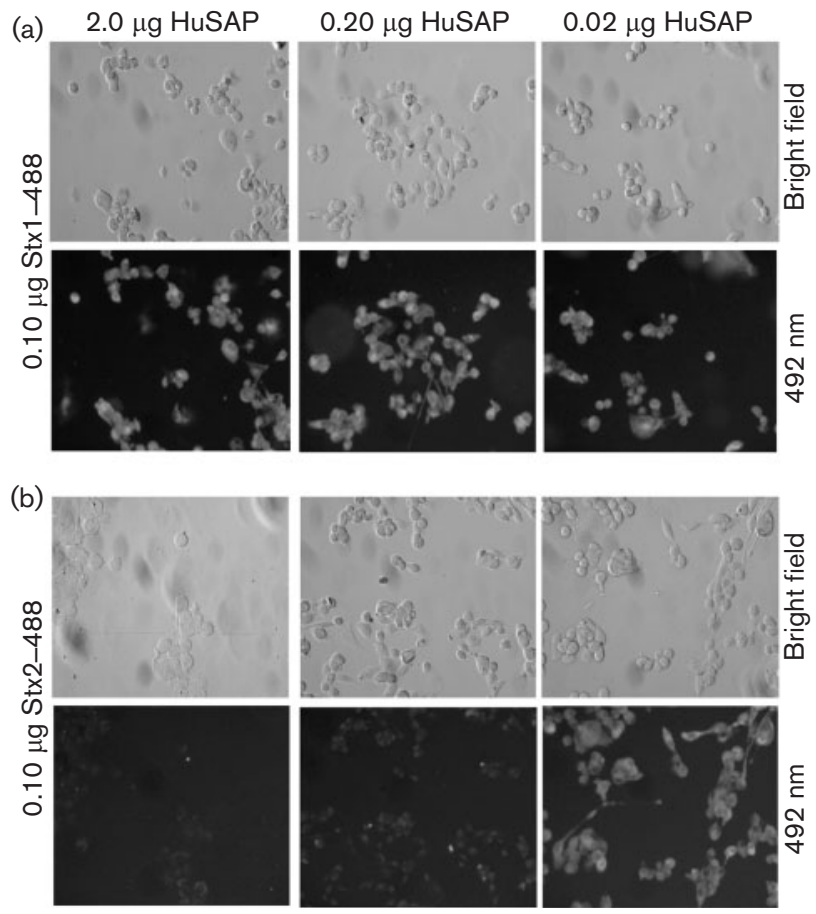

Fig. 2. Binding of $0.10 \mu \mathrm{g}$ Stx1-488 (a) or Stx2-488 (b) to semiconfluent $\mathrm{ACHN}$ renal epithelial cell monolayers following preincubation with decreasing concentrations of $\operatorname{HuSAP}(2.0,0.2$ and $0.02 \mu \mathrm{g})$. Cells were visualized by confocal microscopy. 
(a)

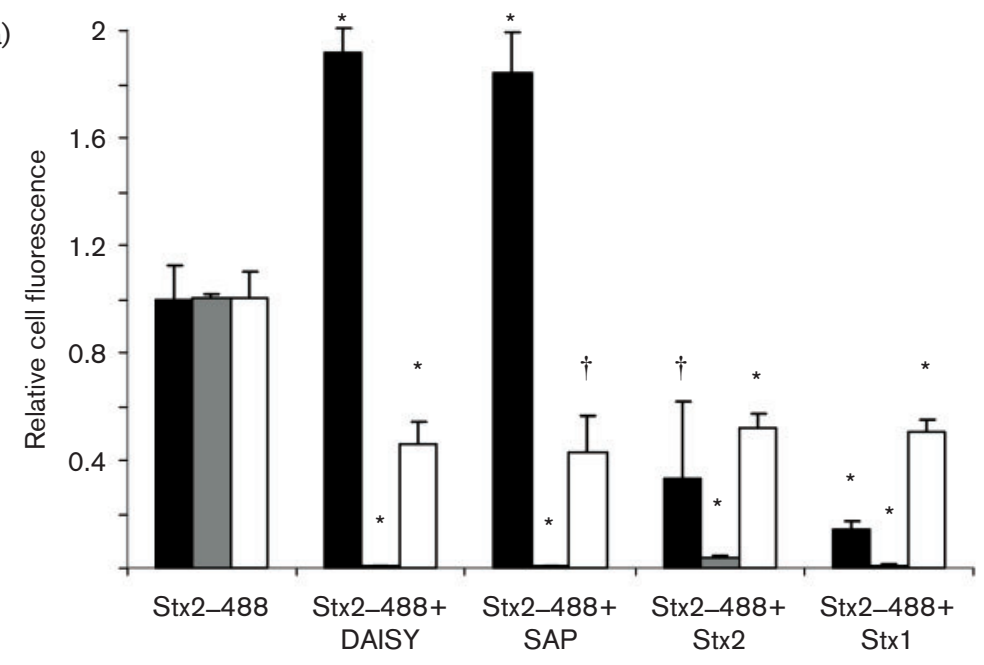

(b)
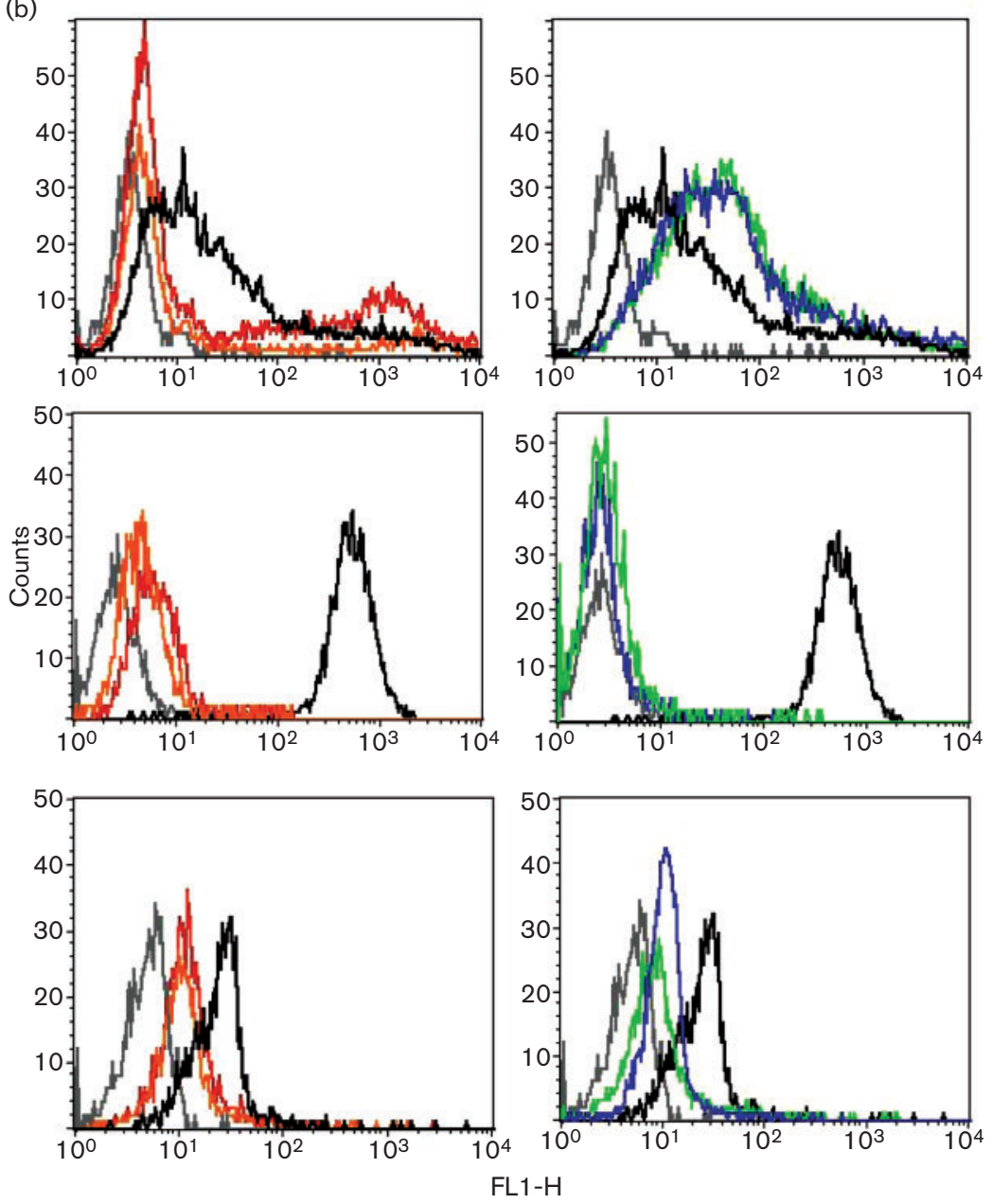

Fig. 3. (a) Binding of $3 \mu \mathrm{g} \mathrm{AF} 488$-labelled Stx2 to human neutrophils (black bars), Ramos B cells (grey bars) and murine neutrophils (white bars) following pre-incubation with 100fold (w/w) $\mathrm{Gb}_{3}$ receptor analogue DAISY, 40fold (w/w) HuSAP or 35 -fold (w/w) unlabelled Stx1 or Stx2 in PBS. Following washing with PBS, the mean fluorescence of the entire cell population was determined by flow cytometry. Data are presented as the mean fluorescence relative to Stx2-488 alone for each cell type. This value was 32.2 relative fluorescent units (RFU) for human neutrophils, 24.79 RFU for murine neutrophils and 739.4 RFU for Ramos cells. Error bars represent the SD about the means of three independent observations. Significant differences (Student's $t$-test) compared with Stx2-488 alone are indicated: *, $P \leqslant 0.002 ; \quad \dagger, P<0.05$. (b) Representative histograms of fluorescence (FL1 channel fluorescent intensity, $x$-axis) of cell populations (cell number, $y$-axis) for human neutrophils (top), Ramos cells (middle) and murine neutrophils (bottom). The left panel of each displays negative-control cells (grey lines), St×2-488 treated cells (black lines), and Stx2-488-treated cells following pre-incubation with unlabelled Stx2 (red lines) or Stx1 (orange lines). The right panel displays untreated and Stx2-488-treated cells, and Stx2-488- plus DAISY-treated cells (green lines) and Stx2-488- plus HuSAP-treated cells (blue lines).
To explore these differences in Stx 2 binding to ACHN cells and neutrophils in more detail, we repeated the Stxbinding inhibition assays using DAISY instead of HuSAP (Fig. 3). DAISY is a decavalent $\mathrm{Gb}_{3}$-related Stx receptor analogue that inhibits the activity of Stx1 and Stx2 in a Vero cytotoxicity assay, as well as protecting mice from a lethal dose of these toxins (Mulvey et al., 2003). In these experiments, DAISY, like HuSAP, increased the binding of Stx2-488 to human neutrophils by $91.6 \% \quad(P<0.002$, Student's $t$-test). In contrast, Stx2-488 binding to Ramos cells, a $\mathrm{Gb}_{3}$-expressing non-adherent target cell line, was completely inhibited by DAISY. The failure of DAISY to inhibit Stx2 binding to human neutrophils, which do not express $\mathrm{Gb}_{3}$ or $\mathrm{Gb}_{3}$-related glycolipids (Macher \& Klock 
1980; Fukuda et al., 1985), confirmed that Stx2 binds to these cells by an alternative $\mathrm{Gb}_{3}$-independent receptor mechanism. However, both HuSAP and DAISY inhibited specific Stx2-488 binding to murine neutrophils (Fig. 3). Stx2-488, therefore, appears to bind to murine neutrophils by a classical $\mathrm{Gb}_{3}$-dependent, HuSAP- and DAISYinhibitable mechanism. Similar $\mathrm{Gb}_{3}$-dependent binding by Stx has been described to both porcine (Winter et al., 2004) and ovine (Menge et al., 2006) neutrophils. Moreover, the data presented in Fig. 3 also demonstrated that excess unlabelled Stx2, as well as unlabelled Stx1, inhibited Stx2-488 binding to all three cell types. The implication of this data is that Stx1 and Stx2 bind to the same or similar $\mathrm{Gb}_{3}$-independent receptors on human but not murine neutrophils.

As Stx 1 and Stx2 bind to $\mathrm{Gb}_{3}$ exclusively via their B subunit pentamers, we investigated binding of the FPLC-separated Stx2 A and B subunits to neutrophils. The data in Fig. 4 suggested that the Stx2 A subunit is largely responsible for the $\mathrm{Gb}_{3}$-independent binding of the Shiga toxins to human neutrophils. The ability of excess unlabelled Stx 2 holotoxin to inhibit the binding of the AF-labelled A subunit to human neutrophils provided evidence for the specificity of this interaction. To the best of our knowledge, this represents the first report of A subunit-mediated binding of the Shiga toxins to a eukaryotic cell. Specific binding of the Stx2 B subunit was also observed, suggesting the possibility of multiple Stx2 receptors on the human neutrophil surface. This was confirmed with both FPLCpurified and recombinant Stx B subunit pentamer preparations. Stx2 binding to murine neutrophils was exclusive to the B subunit pentamer (Fig. 4), an

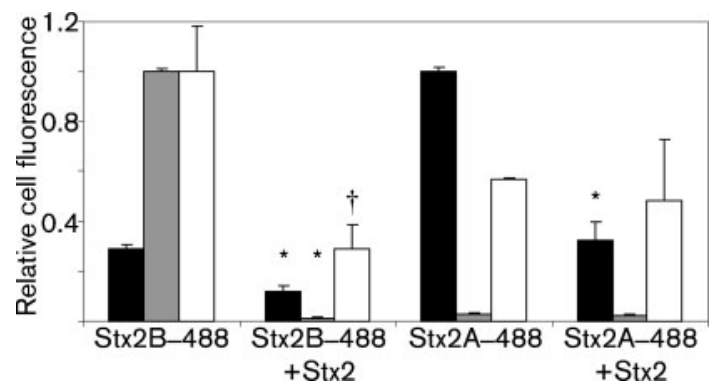

Fig. 4. Binding of $1 \mu \mathrm{g} A F$ 488-labelled purified Stx2 A and $B$ subunits to human neutrophils (black bars), Ramos B cells (grey bars) or murine neutrophils (white bars) and inhibition with 50-fold $(w / w)$ unlabelled Stx2 holotoxin. Following washing with PBS, the mean fluorescence of the entire cell population was determined by flow cytometry. Data are presented as mean fluorescence relative to the dominant subunit fluorescence for each cell type. This value was 787.31 RFU for Stx2 A-488-treated human neutrophils, 591.01 RFU for Stx2 B-488-treated Ramos cells and 65.2 RFU for Stx2 B-488-treated murine neutrophils. Error bars represent the SD about the means of four independent observations. Significant differences (Student's $t$-test) compared with Stx2 A488 or Stx2 B-488 alone are indicated: *, $P \leqslant 0.002 ; \dagger, P<0.05$. observation that again is consistent with the conclusion that Stx2 binding to murine neutrophils occurs as a result of a classical DAISY-inhibitable, $\mathrm{Gb}_{3}$-dependent binding interaction (Fig. 3).

In a previous article, we demonstrated how a DAISYrelated compound, called STARFISH, formed back-to-back complexes with the Stx1 B subunit pentamer (Kitov et al., 2000). As a consequence, DAISY-mediated complex formation should serve to increase the total number of Stx2 molecules bound to human neutrophils using a binding domain on a single A subunit of the Stx2-DAISY complex. The data presented in Fig. 3 are entirely consistent with this suggestion. The increased binding of Stx2 to human neutrophils in the presence of HuSAP (Fig. 3) might also be explained by the formation of Stx2HuSAP complexes. Although the enhanced binding of Stx2 to human neutrophils in the presence of DAISY and HuSAP might also have occurred if these agents were capable of inducing the presentation of a greater number of receptors on the surface of these cells, the near-identical behaviour of these two biochemically distinct inhibitors in enhancing Stx2 binding to human neutrophils suggests that complex formation is the more likely of the two possibilities.

\section{Shiga toxin transfer to target cells}

In their report, te Loo et al. (2000) demonstrated that human neutrophils were capable of transferring their cargo of Stx to glomerular microvascular endothelial cells in vitro. They also demonstrated that Stx binding did not trigger toxin internalization in these cells. The data presented in Fig. 5 confirmed these results. The data also demonstrated that HuSAP is capable of inhibiting the transfer of Stx2-488 from human neutrophils to a target cell containing $\mathrm{Gb}_{3}$ receptors, whereas uninhibited Stx2488 transfer occurs in tissue culture medium supplemented with $10 \%$ FBS, which contains bovine SAP. In addition, incubating the toxin with HuSAP prior to adding it to neutrophils and subsequently removing the unbound complexes yielded identical results, the difference between the two datasets being insignificant $(P=0.523$, Student's $t$ test), further illustrating the ability of Stx2 to engage HuSAP and its $\mathrm{Gb}_{3}$-independent receptor simultaneously on human neutrophils. The implication of the observation in our previous report that DAISY failed to inhibit Stx2 binding to HuSAP, together with the results in Fig. 5, is that Stx2 contains three separate non-overlapping binding domains for HuSAP, DAISY and its $\mathrm{Gb}_{3}$-independent receptor on human neutrophils.

The data in Fig. 6 support this conclusion. These results demonstrated that pre-loading human neutrophils with unlabelled Stx 2 significantly $(P \leqslant 0.002$, Student's $t$-test $)$ increased their capacity to bind HuSAP-488 by $103.7 \%$. In contrast, pre-loading human neutrophils with unlabelled Stx1 only increased their capacity for binding HuSAP-488 by $35.4 \%$, a statistically insignificant result $(P>0.05$, 


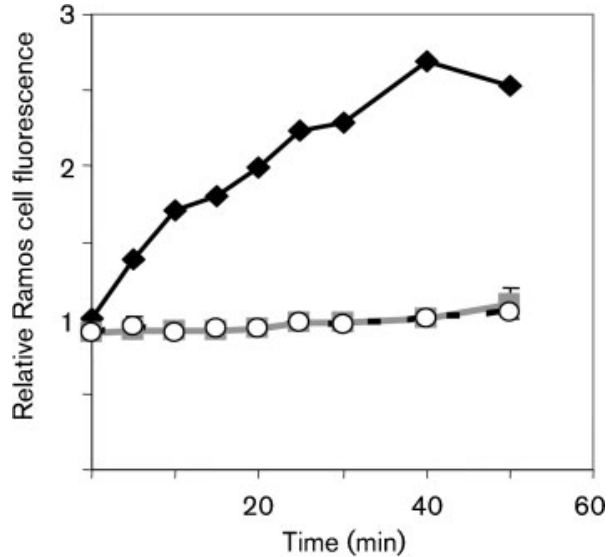

Fig. 5. Transfer of Stx2-488 bound to the surface of human neutrophils to Ramos cells in RPMI $1640+10 \%$ HI-FBS. Following incubation with Stx2-488, the human neutrophils were washed to remove unbound toxin and then incubated with Ramos cells. Samples were withdrawn every $5 \mathrm{~min}$ and the mean fluorescence of the Ramos cell population determined by flow cytometry. Black line $+\boldsymbol{\nabla}$, transfer of Stx2-488 to Ramos cells in the absence of HuSAP added to the transfer medium; grey line $+\square$, transfer of Stx2-488 to Ramos cells in the presence of HuSAP added to the transfer medium; dashed line $+\bigcirc$, HuSAP was mixed with Stx2-488 prior to adding these complexes to human neutrophils, after washing with PBS to remove unbound HuSAP/Stx2-488 complexes, the HuSAP/Stx2-488-loaded neutrophils were added to the Ramos cells. Data are presented as mean fluorescence relative to HuSAP-488 alone cell fluorescence. This value was 18.1 RFU. Error bars represent the SD about the means of three independent observations.

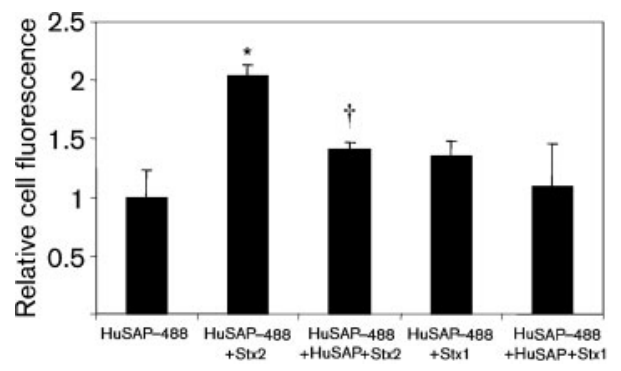

Fig. 6. (a) Binding of AF 488-labelled HuSAP to human neutrophils following a 30 min pre-incubation with $3 \mu \mathrm{g}$ Stx 1 or Stx2 or no toxin control with and without a 10 -fold (w/w) excess of unlabelled HuSAP. Following washing with PBS, the mean fluorescence of the entire cell population was determined by flow cytometry. Error bars represent the SD about the means of three independent observations. Data are presented as mean fluorescence relative to HuSAP-488 alone cell fluorescence. This value was 27.33 RFU. A significant difference compared with HuSAP488 alone $\left({ }^{*}, P \leqslant 0.002\right.$, Student's $t$-test) and a significant reduction compared with HuSAP-488+Stx2 $(\dagger, P<0.05)$ are indicated.
Student's $t$-test). Furthermore, the addition of a 10 -fold excess of unlabelled HuSAP significantly $(P<0.05$, Student's $t$-test) reduced the amount of HuSAP-488 bound to Stx2-loaded neutrophils, thereby confirming the specificity of the HuSAP-488/Stx2 binding interaction. These results demonstrated that Stx2 can simultaneously bind to both HuSAP and human neutrophils in circulation. However, the inclusion of excess unlabelled HuSAP failed to reduce the binding of HuSAP-488 to Stx2-loaded neutrophils to a value less than the mean fluorescence observed when HuSAP-488 was incubated with neutrophils that had not been exposed to Stx2 (Fig. 6). This suggests that HuSAP-488 binding to human neutrophils in the absence of Stx2 probably represents non-specific binding.

Before neutrophils enter the post-glomerular venules, they are forced, because of their physical size, to squeeze through the narrow glomerular capillaries (Downey et al., 1990). This slows their progress considerably, regardless of their state of activation, and brings the neutrophil surfaces into very close contact with the $\mathrm{Gb}_{3}$-bearing endothelial cells of the glomerular capillaries (Kuligowski et al., 2006). We have shown that HuSAP binds to Stx2 on human neutrophils (Fig. 6) and prevents its transfer to target cells (Fig. 5). Given the physical constraints imposed by the diameter of these capillaries, HuSAP may not be able to effectively prevent the exchange of Stx2 between the two closely opposed membrane-associated receptor systems in the glomerular capillaries.

Bitzan et al. (1994) used direct and indirect immunofluorescence procedures to conclude that Stx1 and Stx2, as well as the variant forms of Stx2, bind to PBS-washed human erythrocytes in $\mathrm{Gb}_{3}$-dependent manner. However, we have demonstrated that HuSAP inhibits $\mathrm{Gb}_{3}$-dependent Stx2 binding to different cell types (Figs 2 and 3 ). It is therefore unlikely that significant Stx2 binding to erythrocytes would have been detected by Bitzan et al. (1994) if their experiments had been conducted using whole blood containing HuSAP. Depending on P blood group phenotype, erythrocytes might well represent a 'non-pathological sink' for Stx1 but not, in the presence of physiological concentrations of HuSAP, for Stx2. The greater association between Stx 2 and HUS in humans might be partially related, therefore, to the toxin's exclusive association, in the presence of HuSAP, with $\mathrm{Gb}_{3}$-independent receptors on human neutrophils. This is consistent with the observation that Stx2 binds preferentially to neutrophils in subjects infected with E. coli O157:H7 (te Loo et al., 2001a).

\section{Conclusions}

The results presented herein are consistent with the conclusion that Stx2, and probably Stx1 (Fig. 3), bind to a $\mathrm{Gb}_{3}$-independent receptor expressed on human but not murine neutrophils in vitro. Our findings suggest, therefore, that murine models may not be appropriate for 
studying the role of neutrophils in the Stx-associated complications of E. coli $\mathrm{O} 157: \mathrm{H} 7$ infections in humans. As suggested by the results in our previous article (Armstrong et al., 2006), the protective effect of HuSAP in the murine model probably depends on its ability to form multimeric complexes with Stx2, which, because of their inability to bind to murine neutrophils or, for that matter, other cell types in blood, may divert the toxin into a non-lethal catabolic pathway. As HuSAP does not prevent Stx2 binding to human neutrophils, similar protection may not occur in humans suffering from an EHEC infection. Inhibiting Stx2 binding to human neutrophils may represent a novel therapeutic approach to preventing the extraintestinal complications related to EHEC infections. Also, the finding that HuSAP may enhance Stx 2 binding to human neutrophils through the formation of complexes could provide an explanation for why EHEC strains expressing both Stx1 and Stx2 or Stx2 alone are more likely to cause HUS than strains expressing only Stx1 (Beutin et al., 2004). The observation that multivalent $\mathrm{Gb}_{3}$ analogues such as DAISY may also increase Stx2 binding to human neutrophils in blood, thereby potentially intensifying the delivery of the toxin to its extraintestinal target organs, suggest that caution should now be exercised in proposing human clinical trials with such drugs.

\section{ACKNOWLEDGEMENTS}

This work was supported by operating grants MWS 56081 from the Canadian Institutes for Health Research (CIHR) to G. D. A. and a Province of Alberta Graduate Award (T.G.). The authors declare no financial conflict of interest. We thank Dr M. B. Pepys for supplying us with the purified HuSAP, as well as his advice concerning its use, and Ms Adrienne Ma, Mr Bryan Heit and Dr Paul Kubes for their assistance in isolating the human and murine neutrophils.

\section{REFERENCES}

Armstrong, G. D., Mulvey, G. L., Marcato, P., Griener, T. P., Kahan, M. C., Tennent, G. A., Sabin, C. A., Chart, H. \& Pepys, M. B. (2006). Human serum amyloid $\mathrm{P}$ component protects against Escherichia coli O157:H7 Shiga toxin 2 in vivo: therapeutic implications for hemolytic-uremic syndrome. J Infect Dis 193, 1120-1124.

Beutin, L., Krause, G., Zimmermann, S., Kaulfuss, S. \& Gleier, K. (2004). Characterization of Shiga toxin-producing Escherichia coli strains isolated from human patients in Germany over a 3-year period. J Clin Microbiol 42, 1099-1108.

Bitzan, M., Richardson, S., Huang, C., Boyd, B., Petric, M. \& Karmali, M. A. (1994). Evidence that Verotoxins (Shiga-like toxins) from Escherichia coli bind to P blood group antigens of human erythrocytes in vitro. Infect Immun 62, 3337-3347.

Brigotti, M., Caprioli, A., Tozzi, A. E., Tazzari, P. L., Ricci, F., Conte, R., Carnicelli, D., Procaccino, M. A., Minelli, F. \& other authors (2006). Shiga toxins present in the gut and in the polymorphonuclear leukocytes circulating in the blood of children with hemolytic-uremic syndrome. J Clin Microbiol 44, 313-317.

Cherla, R. P., Lee, S. Y. \& Tesh, V. L. (2003). Shiga toxins and apoptosis. FEMS Microbiol Lett 228, 159-166.
Downey, G. P., Doherty, D. E., Schwab, B., III, Elson, E. L., Henson, P. M. \& Worthen, G. S. (1990). Retention of leukocytes in capillaries: role of cell size and deformability. J Appl Physiol 69, 1767-1778.

Flagler, M. J., Strasser, J. E., Chalk, C. L. \& Weiss, A. A. (2007). Comparative analysis of the abilities of Shiga toxins 1 and 2 to bind to and influence neutrophil apoptosis. Infect Immun 75, 760-765.

Fraser, M. E., Fujinaga, M., Cherney, M. M., Melton-Celsa, A. R., Twiddy, E. M., O'Brien, A. D. \& James, M. N. (2004). Structure of Shiga toxin type 2 (Stx2) from Escherichia coli O157:H7. J Biol Chem 279, 27511-27517.

Fukuda, M. N., Dell, A., Oates, J. E., Wu, P., Klock, J. C. \& Fukuda, M. (1985). Structures of glycosphingolipids isolated from human granulocytes. The presence of a series of linear poly- $N$-acetyllactosaminylceramide and its significance in glycolipids of whole blood cells. J Biol Chem 260, 1067-1082.

Geelen, J. M., van der Velden, T. J., Te Loo, D. M., Boerman, O. C., van den Heuvel, L. P. \& Monnens, L. A. (2007). Lack of specific binding of Shiga-like toxin (Verocytotoxin) and non-specific interaction of Shiga-like toxin 2 antibody with human polymorphonuclear leucocytes. Nephrol Dial Transplant 22, 749-755.

Hawkins, P. N., Tennent, G. A., Woo, P. \& Pepys, M. B. (1991). Studies in vivo and in vitro of serum amyloid $\mathrm{P}$ component in normals and in a patient with AA amyloidosis. Clin Exp Immunol 84, 308-316.

Head, S. C., Karmali, M. A. \& Lingwood, C. A. (1991). Preparation of VT1 and VT2 hybrid toxins from their purified dissociated subunits. Evidence for B subunit modulation of a subunit function. J Biol Chem 266, 3617-3621.

Kaplan, B. S., Meyers, K. E. \& Schulman, S. L. (1998). The pathogenesis and treatment of hemolytic uremic syndrome. J Am Soc Nephrol 9, 1126-1133.

Karmali, M. A., Petric, M., Lim, C., Fleming, P. C. \& Steele, B. T. (1983). Escherichia coli cytotoxin, haemolytic-uraemic syndrome, and haemorrhagic colitis. Lancet 2, 1299-1300.

Kimura, T., Tani, S., Matsumoto Yi, Y. \& Takeda, T. (2001). Serum amyloid $\mathrm{P}$ component is the Shiga toxin 2-neutralizing factor in human blood. J Biol Chem 276, 41576-41579.

Kitov, P. I., Sadowska, J. M., Mulvey, G., Armstrong, G. D., Ling, H., Pannu, N. S., Read, R. J. \& Bundle, D. R. (2000). Shiga-like toxins are neutralized by tailored multivalent carbohydrate ligands. Nature $\mathbf{4 0 3}$, 669-672.

Kuligowski, M. P., Kitching, A. R. \& Hickey, M. J. (2006). Leukocyte recruitment to the inflamed glomerulus: a critical role for plateletderived P-selectin in the absence of rolling. J Immunol 176, 69916999.

Liu, J., Akahoshi, T., Sasahana, T., Kitasato, H., Namai, R., Sasaki, T., Inoue, M. \& Kondo, H. (1999). Inhibition of neutrophil apoptosis by Verotoxin 2 derived from Escherichia coli O157 : H7. Infect Immun 67, 6203-6205.

Macher, B. A. \& Klock, J. C. (1980). Isolation and chemical characterization of neutral glycosphingolipids of human neutrophils. J Biol Chem 255, 2092-2096.

Marcato, P., Vander Helm, K., Mulvey, G. L. \& Armstrong, G. D. (2003). Serum amyloid $P$ component binding to Shiga toxin 2 requires both A subunit and B pentamer. Infect Immun 71, 60756078.

Menge, C., Eisenberg, T., Stamm, I. \& Baljer, G. (2006). Comparison of binding and effects of Escherichia coli Shiga toxin 1 on bovine and ovine granulocytes. Vet Immunol Immunopathol 113, 392-403.

Mulvey, G., Vanmaele, R., Mrazek, M., Cahill, M. \& Armstrong, G. (1998). Affinity purification of Shiga-like toxin I and Shiga-like toxin II. J Microbiol Methods 32, 247-252. 
Mulvey, G. L., Marcato, P., Kitov, P. I., Sadowska, J., Bundle, D. R. \& Armstrong, G. D. (2003). Assessment in mice of the therapeutic potential of tailored, multivalent Shiga toxin carbohydrate ligands. $J$ Infect Dis 187, 640-649.

Nataro, J. P. \& Kaper, J. B. (1998). Diarrheagenic Escherichia coli. Clin Microbiol Rev 11, 142-201.

Taguchi, T., Uchida, H., Kiyokawa, N., Mori, T., Sato, N., Horie, H., Takeda, T. \& Fujimoto, J. (1998). Verotoxins induce apoptosis in human renal tubular epithelium derived cells. Kidney Int 53, 1681-1688.

Tazzari, P. L., Ricci, F., Carnicelli, D., Caprioli, A., Tozzi, A. E., Rizzoni, G., Conte, R. \& Brigotti, M. (2004). Flow cytometry detection of Shiga toxins in the blood from children with hemolytic uremic syndrome. Cytometry B Clin Cytom 61, 40-44.

te Loo, D. M., Monnens, L. A., van Der Velden, T. J., Vermeer, M. A., Preyers, F., Demacker, P. N., van Den Heuvel, L. P. \& van Hinsbergh,
V. W. (2000). Binding and transfer of Verocytotoxin by polymorphonuclear leukocytes in hemolytic uremic syndrome. Blood 95, 33963402.

te Loo, D. M., Heuvelink, A. E., de Boer, E., Nauta, J., van der Walle, J., Schroder, C., van Hinsbergh, V. W., Chart, H., van de Kar, N. C. \& van den Heuvel, L. P. (2001a). Vero cytotoxin binding to polymorphonuclear leukocytes among households with children with hemolytic uremic syndrome. J Infect Dis 184, 446-450.

te Loo, D. M., van Hinsbergh, V. W., van den Heuvel, L. P. \& Monnens, L. A. (2001b). Detection of Verocytotoxin bound to circulating polymorphonuclear leukocytes of patients with hemolytic uremic syndrome. J Am Soc Nephrol 12, 800-806.

Winter, K. R., Stoffregen, W. C. \& Dean-Nystrom, E. A. (2004). Shiga toxin binding to isolated porcine tissues and peripheral blood leukocytes. Infect Immun 72, 6680-6684. 